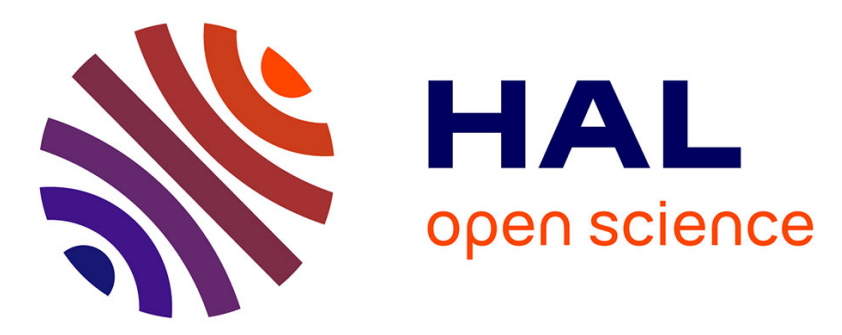

\title{
Special issue on Ground robots operating in dynamic, unstructured and large-scale outdoor environments
}

\author{
Simon Lacroix, Frank Schneider, Dennis Wildermuth, Alan Winfield
}

\section{To cite this version:}

Simon Lacroix, Frank Schneider, Dennis Wildermuth, Alan Winfield. Special issue on Ground robots operating in dynamic, unstructured and large-scale outdoor environments: Editorial. Journal of Field Robotics, 2015, 32 (4), 10.1002/rob.21604 . hal-01522325

\section{HAL Id: hal-01522325 \\ https://hal.science/hal-01522325}

Submitted on 14 May 2017

HAL is a multi-disciplinary open access archive for the deposit and dissemination of scientific research documents, whether they are published or not. The documents may come from teaching and research institutions in France or abroad, or from public or private research centers.
L'archive ouverte pluridisciplinaire HAL, est destinée au dépôt et à la diffusion de documents scientifiques de niveau recherche, publiés ou non, émanant des établissements d'enseignement et de recherche français ou étrangers, des laboratoires publics ou privés. 
Journal of Field Robotics (JFR)

\section{Special issue on Ground robots operating in dynamic, unstructured and large-scale outdoor environments}

\section{Editorial}

Real-world outdoor applications of ground robots have, to date, been limited primarily to remote inspection of suspected explosive devices and, with less success, to the broader domain of remote survey and inspection in hazardous environments. Such robots have almost exclusively been tele-operated. Also notable as examples of outdoor ground robots are the planetary rovers, currently deployed with great success on the surface of Mars. But with the rapid development of autonomous (driverless) cars, and the emergence of robotic vehicles in agriculture, it is likely that there will be significant growth in both the numbers and scope of commercial ground robots in outdoor environments in the near future.

Applications in real-world outdoor environments demand many different capabilities of robotic systems: from autonomy, decision-making and sensing to robustness, reliability and the ability to manage limitations on communications and power. Outdoor robotic contests such as the European Land Robot Trial (ELROB) or the euRathlon competition present challenges in which participants compete in real world scenarios under realistic conditions and in real terrains, which have not been specially prepared beforehand and are unknown to the competing teams. Example scenarios include autonomous navigation over several kilometres, environmental survey or search and rescue in terrains with dense forests, hilly landscapes with scattered and varied vegetation and water ponds - day and night and with unpredictable weather conditions.

For this special issue we called for papers that present land robot systems deployed in the field in similar realistic challenges. We sought papers that focus on any aspect of robotic systems, from vehicle design to the overall system architecture and control, via terrain mapping, localization, mission planning and execution - with an emphasis on systems that fulfil a specific real world task. We specified that robot or system innovations must be supported by extensive field results. Also that field tests must be under realistic and challenging conditions with respect to the terrain type, the scenario to be achieved, and/or the conditions within which the scenarios must be achieved.

A total of eleven papers were submitted to this special issue, and following a rigorous process of anonymous peer-review six have been selected for publication. The papers included in this special issue cover a broad spectrum of the challenges outlined above. Two papers are dedicated to 3D mapping and localization, two present an autonomous navigation system, and two present wholly integrated systems that operate in populated areas. Outlined below, the six papers of this special issue strongly represent the state-of-the-art in this important area of research. 
Kubelka et al. describe a multi-modal data fusion system for state estimation of a mobile skid-steer robot. The system is intended for use in Urban Search and Rescue (USAR) missions, typically involving challenging conditions like slippage, moving obstacles and changing light conditions. In order to cope with such demanding environments a wide variety of proprioceptive and exteroceptive sensor readings is combined in a novel fusion scheme based on an Extended Kalman filter for 6DOF orientation and position estimation. The performance of the approach is demonstrated by extensive indoor and outdoor tests using exact ground truth localization data. As an extra contribution to the robotics community, all datasets, including the ground truth measurements, are released to the public.

The article by Meilland et al. presents a novel method for building dense visual maps of large-scale unstructured environments. The approach is intended for real-time localisation and uses an omni-directional key-frame representation of the environment. Locally this representation is composed of augmented spherical panoramas combining photometric information (RGB), depth information (D) and saliency for all viewing directions at a particular point in space. Based on these spheres synthetic photo-realistic views of captured environments at arbitrary 6D locations can be generated. During online navigation, an efficient direct registration technique is employed to accurately localise a monocular camera navigating within the graph. The robustness of the localisation method is validated through extensive field experiments in challenging unstructured environments, containing vegetation, pedestrians, varying illumination conditions, trams and dense traffic.

In Automatic driving on ill-defined roads: an adaptive, shape-constrained, colour-based method, Labrosse et al. present an adaptive colour-based approach to road following on unmarked and/or geometrically poorly defined roads. Camera-based road detection is a challenging task of significant importance to mobile robot navigation. The method described in this paper uses a single Gaussian density with diagonal covariance matrix to model road pixel colour. Road shape is approximated by a symmetric trapezoid in the image plane, with just two variable parameters. The work includes a systematic comparison of colour spaces, relating performance for data sets with different choices of colour space and for different distribution adaptation rates. The key result is to show that a simple colour classifier using a single multivariate Gaussian with diagonal covariance matrix is sufficient for good road-following performance.

Krüsi et al. also present an autonomous navigation system, whose core is a robust teach and repeat localisation approach, here based on Iterative Closest Point matching with a 3D laser scanner. The system also maintains a sequence of submaps, and integrates a local path planner that generates obstacle avoidance trajectories that are by construction dynamically feasible. Extensive results covering several kilometres in urban and natural environments are shown and analyzed. 
Kuemmerle et al. present a robot that integrates a wide spectrum of localisation, mapping and planning functionalities, and that achieves several kilometres autonomous navigation tasks in urban zones populated by pedestrians. The robot exploits a previously learned map, structured in a topological graph to which are anchored occupancy maps. Online, four planar laser sensors are used for both localization and mapping: the system detects negative obstacles, tracks moving objects, and exploits the laser remission values to discriminate between flat vegetation and concrete. Localization relies on a Monte-Carlo approach initiated by GPS readings, and is particularly robust to the presence of pedestrians and changes in the environment with respect to the learned maps. Planning is done in a three-stage hierarchical approach: at the level of the overall graph, on the occupancy maps, and issuing elementary speed commands, while using on-line information on the environment at each stage. The resulting system yields robust long range and long term navigation - the accompanying video is worth watching.

Walter et al. depict a robotized large size forklift that operates in human workplaces. The system is a thorough integration of more than five years of research and development, that spans a wide spectrum of functionalities from manipulation planning and control to task planning, via visual/Lidar scene analysis and local path planning that accounts for dynamic obstacles. The system is commanded through a multimodal interface and special attention has been paid to the awareness of nearby operators, e.g. by reacting to shouted warnings. The robustness of most functions has been experimentally assessed, and the end-to-end system has been thoroughly tested in mock-up environments and actual loading zones. The overall system approaches an operational level.

While six papers do not constitute the complete spectrum of the state of the art, they exhibit some commonalities and trends that are well worth highlighting. Environment mapping and robot localization of course remain at the core of autonomous operation, and are still a highly active area of research. For this purpose the use of Lidar has become widespread, but this of course does not preclude the use of vision as a complement. As for motion planning solutions, these most often rely on a two-level itinerary/trajectory hierarchy. Notably operation among people represents a frontier that is now being crossed. Alongside interactions with human operators or nearby persons, safety issues are here a major concern. These impact throughout the whole system concept, from platform design to the overall software and planning architecture. In summary, the work presented in this special issue provides valuable insights into the current capabilities of robots operating in large-scale outdoor environments. In our view the commercial success of such autonomous vehicles in near future real-world applications seems increasingly assured.

\section{Acknowledgements}

The guest editors would like to take this opportunity to thank all who have contributed to this issue. We would like to thank to editor-in-chief Sanjiv Singh, for inviting us to edit a special issue on the topic. We are especially grateful to managing editor Sanae Minick for her close guidance and help during the review 
process. And we are indebted to the members of the editorial board whose objective and detailed reviews allowed us to generate an issue with the highquality papers outlined above.

Simon Lacroix acknowledges support through CNRS. Alan Winfield, Dennis Wildermuth and Frank Schneider are grateful for the support of EU FP7 project 'Support Action for a Targeted Intelligent Autonomous Robotics Contest: The European Roboathlon' (euRathlon), grant agreement no 601205.

Guest editors:

Simon Lacroix, LAAS/CNRS, Toulouse.

Frank Schneider and Dennis Wildermuth, Cognitive Mobile Systems Department, Fraunhofer Institute for Communication, Information Processing and Ergonomics FKIE, Wachtberg.

Alan Winfield, Bristol Robotics Laboratory, University of the West of England, Bristol.

January 2015 